\title{
Impeller Submergence Depth for Stirred Tanks
}

\author{
Thiyam Thamphasana Devi ${ }^{1}$, Annu Priya Sinha ${ }^{2}$, Meena Thakre ${ }^{2}$, and Bimlesh Kumar $1^{*}$ \\ ${ }^{1}$ Department of Civil Engineering, Indian Institute of Technology Guwahati, Guwahati 781039, India \\ ${ }^{2}$ Agriculture Engineering, JNKVV, Jabalpur, India
}

Received: 4th March 2011; Revised: 12 ${ }^{\text {nd }}$ July 2011; Accepted: 14th July 2011

\begin{abstract}
Impeller submergence governs the performance of mixing tanks employed in oxygen transfer operation. Present work experimentally investigates the effect of impeller submergence depths on oxygen transfer and corresponding power consumption. It has been found that at higher range of impeller submergence, mixing tanks consume less power and gives higher values of oxygen transfer coefficient. Optimal range of submergence depth is 0.7 to 0.9 times the impeller diameter. Copyright (O2011 BCREC UNDIP. All rights reserved.
\end{abstract}

Keywords: oxygen transfer; power consumption; rotational speed; submerged depth; stirred tanks; two-film theory

How to Cite: T.T. Devi, A.P. Sinha, M. Thakre, and B. Kumar. (2011). Impeller Submergence Depth for Stirred Tanks. Bulletin of Chemical Reaction Engineering \& Catalysis, 6 (2): 123-128

\section{Introduction}

Stirred tanks are among the most commonly used pieces of equipment in the chemical and biochemical processes. Oxygen transfer rate and the corresponding power requirement to operate the impeller are vital for design of stirred tanks. The physical and chemical processes taking place in the stirred tank are complex and closely coupled to the underlying transport processes, in particular-the flow field. Therefore, a detailed understanding of the hydrodynamics of stirred tank (velocity field, turbulence, stress field etc.) is useful for optimum design [1-2].

Knowledge about the mixing processes is also important when scaling up a process from laboratory scale to industrial plant scale. The experimental studies of mixing phenomena are usually concentrated around a standard geometry stirred tank where the phases are agitated with some kind of impeller. The study of mixing includes understanding of how gas and liquid phases are transported in the tank, and how this transport is affected by the tank and the impeller(s) geometry, as well as the operational conditions such as stirring rate and pumping capacity. Flow behavior and mixing characteristics in stirred tanks has been studied extensively [1, 311]. The performance of surface aerators is known to depend on the impeller submergence, which indicates the position of impeller in stirred tank $[10,12]$. According to Oldshue [1], there is not only one optimal or unique design for each kind of process. Several designs may satisfy the process specifications [1]. In order to simplify design and minimize costs, standard reactor designs are usually good enough for most processes. Even though the role of impeller submergence has been identified as an important parameter, it has not been systematically investigated. Generally impeller position has been fixed at $1 / 6$ to $1 / 2$ of

* Corresponding Author

E-mail: bimk@iitg.ernet.in, Tel: +91-361-2582420, Fax: +91-361-2582440 
tank diameter [7]. Zlokarnik [13] has found relatively greater effect of the impeller submergence on the oxygen transfer rate than on the power consumption. Backhurst et al [14] has found that the oxygen-transfer rate per unit power consumption was optimum at an impeller clearance of twice the impeller diameter and that, with a further increase in impeller clearance, the value of oxygen-transfer rate per unit power consumption decreased. Takase et al [15] has reported different effects of impeller clearance. Patil et al [10] has conformed the observations reported by Backhurst et al [14]. For a pitched blade down flow turbine (PBTD) and standard disk turbine (DT), optimality has been observed when an impeller clearance is twice the impeller diameter [10].

The economics of stirred tanks, particularly operating costs, are contributing much more heavily to system selection [16-17]. Because of the widespread applications of stirred tanks in the process industry, even a small reduction in the operational costs of these vessels can result in major cost savings. It is essential that the design and operation of stirred tank must be as near as is possible or practicable. The design guideline on Rushton turbine (generally called as standard turbine) is well documented in the literature, whereas in case of non-standard impeller such as curved blade, arrowhead impeller are not always easy to find in the open literature. Present work tries to find out the optimal impeller submergence for non-standard impeller (curved blade impeller). The effect of impeller position has been investigated in the present work for optimal oxygen transfer and power consumption.

\section{Materials and Method}

Experiment has been carried out with an objective of finding the effect of impeller position on oxygen transfer coefficient and power consumption. Two sizes of unbaffled stirred tanks $\left(\mathrm{A}=0.0314 \mathrm{~m}^{2}\right.$ and $\left.0.01766 \mathrm{~m}^{2}\right)$ have been used in the experimentation. Fig. 1 shows schematic diagram of the setup used in the present study. The various geometric dimensions of the aerator are: D (tank diameter), $\mathrm{H}$ (water depth), $\mathrm{h}$ (impeller submergence or distance between the horizontal bottom of the tank and the top of the blades) and d (diameter of the impeller). Diameter of the tanks (D) is $200 \mathrm{~mm}$ and $150 \mathrm{~mm}$, respectively. Standard geometry of impeller (Rushton turbine) has been documented well in the literature [11]. Thus the impeller used in the present study is six curved blade impeller
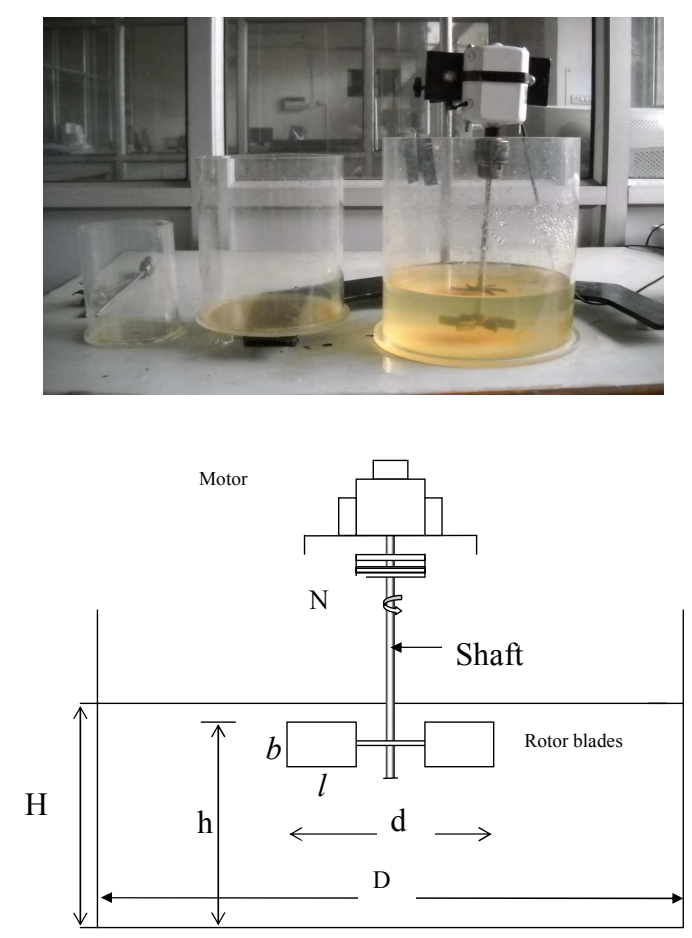

Figure 1. Experimental setup and Schematic diagram

Given the same conditions of power per unit volume, curved blade impeller is having higher value of mass transfer (www.chemineer.com) than conventional flat blades disc impeller (Rushton turbine). When a single impeller is to be used, a liquid level equal to the diameter is optimum, with the impeller located at the center for an allliquid system $[7,18]$.

Generally unbaffled tanks are being employed for surface aerators, because unbaffled tanks give rise to higher fluid-particle mass transfer rates for a given power consumption [4], which is the paramount importance in designing aeration system. Baffled tanks are also giving rise to dead zones, actually worsening the mixing performance of an aeration system [7]. It has been also recognized that the local value of the mass transfer rates would probably vary from one region to another in baffled tanks [19]. There are regions in the tanks where the surface renewal rate is high and the other parts where the liquid is relatively stationary [19]. This non-uniformity of local value of mass transfer rates is the fundamental disadvantage of the use of baffled tank in mass transfer process.

\subsection{Power Consumption}

Power consumption is a basic integral quantity 


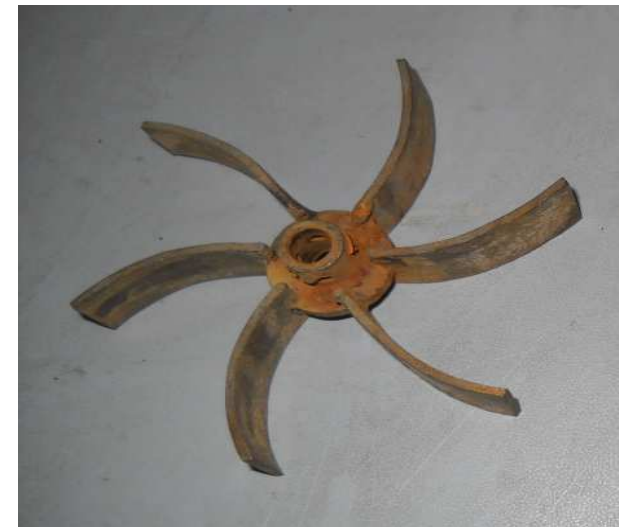

Figure 2. Impeller Used in the Experiments

Table 1. Impeller geometrical properties

\begin{tabular}{cccc}
\hline $\begin{array}{c}\text { Impeller } \\
\text { diameter } \\
(\mathrm{d})(\mathrm{mm})\end{array}$ & $\begin{array}{c}\text { Blade } \\
\text { length } \\
(\mathrm{mm})\end{array}$ & $\begin{array}{c}\text { Blade } \\
\text { width } \\
(\mathrm{mm})\end{array}$ & $\begin{array}{c}\text { Blade } \\
\text { thickness } \\
(\mathrm{mm})\end{array}$ \\
\hline 80 & 20 & 16 & 0.8 \\
60 & 15 & 12 & 0.6 \\
45 & 11.25 & 9 & 0.45 \\
40 & 10 & 8 & 0.4 \\
30 & 7.5 & 6 & 0.3 \\
\hline
\end{tabular}

in an aerator, which determines the other processes involved in aeration phenomena such as 'mass transfer rates', 'gas hold up' etc [20-21]. The power usage in mass transfer operations is very important in judging the aeration performance of the aerator. Power draw is defined as the amount of energy necessary in a period of time, in order to generate the movement of the fluid within a container (e.g. bioreactor, mixing tank, chemical reactor etc.) by means of mechanical or pneumatic agitation [22]. Power draw in systems has been measured by means of a wattmeter. The power available at the shaft was calculated as follows [22]. Let $P_{1}$ and $P_{2}$ are the power requirements under no load and loading conditions at the same speed of rotation. Then the effective power available to the shaft is $P_{\text {eff }}=P_{2}-P_{1}-$ Losses. Reproducibility of measurements has been found to be $\pm 5 \%$. The main advantages of the estimation of power draw by electrical measurements are: (1) It is a simple method, (2) little instrumentation is required and (3) high investment is not required.

\subsection{Oxygen Transfer Rate}

According to two-film theory [23], the oxygen transfer coefficient at $\mathrm{T}{ }^{\circ} \mathrm{C}, K_{L} a_{T}$ may be expressed as follows (Eq. 1):

$$
K_{L} a_{T}=\left[\ln \left(C_{s}-C_{0}\right)-\ln \left(C_{s}-C_{t}\right)\right] / t
$$

where, $\ln$ represents natural logarithm and $C_{s}, C_{0}$ and $C_{t}$ are dissolved oxygen (DO) concentrations in parts per million (ppm), $C_{s}=$ the saturation $\mathrm{DO}$ at time tending to very large values, $C_{0}$ is at the beginning of time $\mathrm{t}=0$ and $C_{t}$ is at time $\mathrm{t}=\mathrm{t}$. The value of $K_{L} a_{T}$ can be obtained as slope of the linear plot between $\ln \left(C_{s}-C_{t}\right)$ and time $t$. The value of $K_{L} a_{T}$ can be corrected for a temperature other than the standard temperature of $20{ }^{\circ} \mathrm{C}$ as $K_{L} a_{20}$, using the Vant- Hoff Arrhenius, Eq. (2):

$$
K_{L} a_{T}=K_{L} a_{20} \mathrm{q}^{(T-20)}
$$

where $\mathrm{q}$ is the temperature coefficient equal to 1.02 for pure water. Once the impeller starts rotating, DO meter (Thermo-Orion $\AA$ ) reading is noted at regular interval up to the point when the DO values reaches $80 \%$ of the saturation value or above. Thus the known values of DO measurements in terms of $C_{t}$ at regular intervals of time $t$ (including the known value of $C_{0}$ at $t=0$ ) a line is fitted, by linear regression analysis of Eq.1, between the logarithm of $\left(C_{s}-C_{t}\right)$ and $t$, by assuming different but appropriate values of $C_{s}$ such that the regression that gives the minimum "standard error of estimate" is taken and thus the values of $K_{L} a_{T}$ and $C_{s}$ has been obtained simultaneously. Oxygen transfer modeling by two-film theory assumes that a single and constant value of $C_{s}$ is adequately representative of the equilibrium DO for the liquid phase oxygen mass transfer for the entire aeration systems and the transfer process is predominately liquid phase mass transfer controlled and the gas phase resistance to transfer can be ignored. Now by fixing the value of $C_{s}$, the value of $K_{L} a_{T}$ has been determined by the best fit straight line, semilogarithmic plot of $\left(C_{s}-C_{t}\right)$ and $t$. It may be noted that the value of $C_{s}$ used in the log-deficit approach can be based on field measurement, published value, or simple assumption [24]. It is a common practice to fix the value of $C_{s}$ around the maximum DO value with some increment in it, as long as it gives the best fit. The slope of such a straight line, semi-logarithmic is equal to $-K_{L} a_{T}$. It is worth noting that selection of a particular value for $C_{\mathrm{s}}$ will influence the resultant value for $K_{L} a_{T}$ determined by this approach. The oxygen transfer coefficient calculated using this method may have errors, because the method used 
requires a straight line to fit the data, the error could be reduced if the linear regression coefficient of the chosen line is high. In that case the slope of the line would accurately represent the oxygen transferred to the water.

\section{Results and Discussion}

The performance of stirred tank is known to depend on the impeller submergence. Therefore, the effect of impeller submergence has been investigated systematically. Fig. 3 illustrates
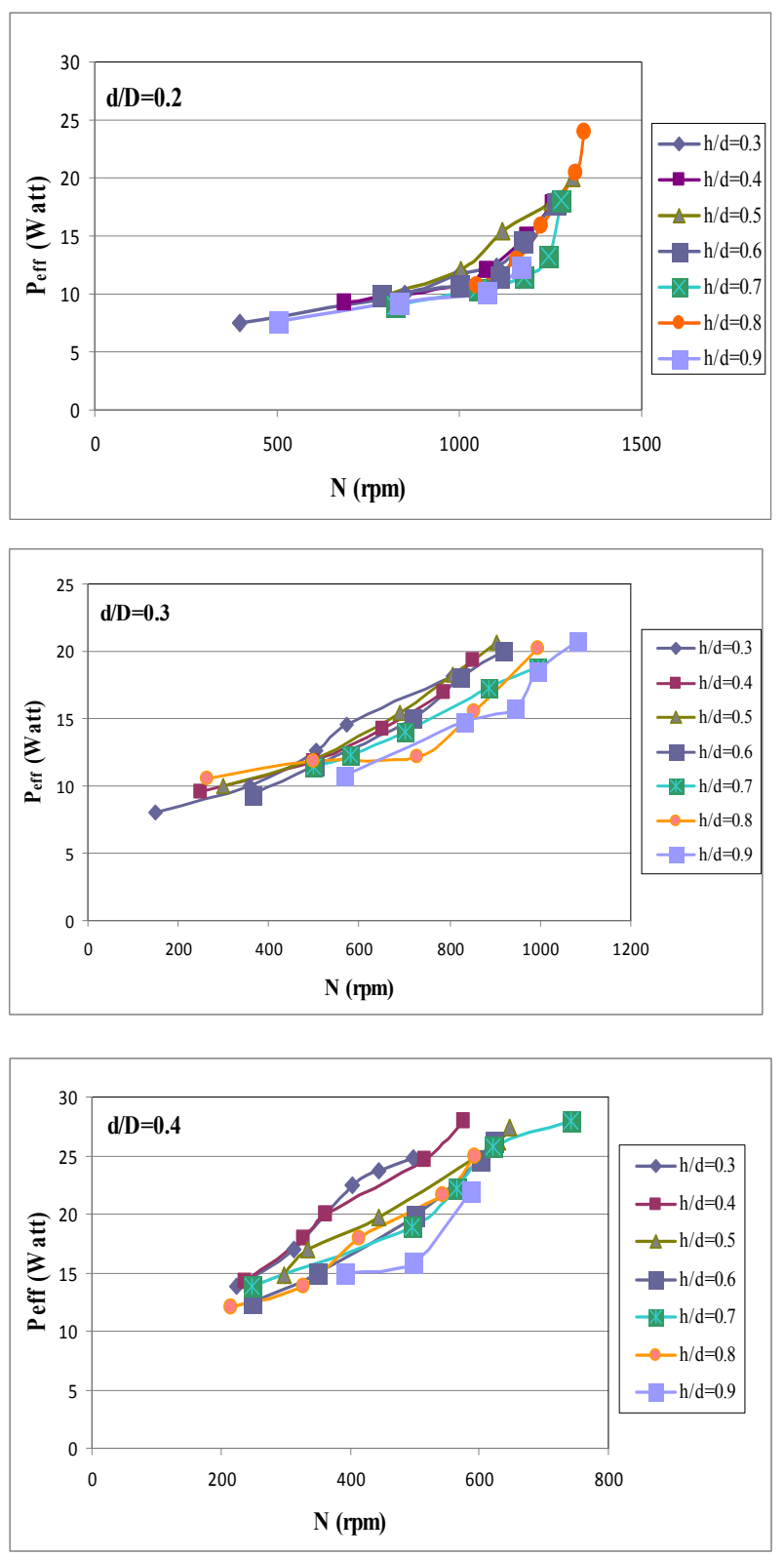

Figure 3a. Influence of submergence depth at power consumption (200mm tank) observations made on power consumption with different submergence depths of impeller. The value of $\mathrm{h} / \mathrm{d}$ varies from 0.3 to 0.9 . Power consumption is strongly dependent on the gas holdup near the impeller i.e., the local gas hold-up in the vicinity of the impeller [25]. As the size of this region increases, impeller rotates in progressively increasing liquid free region thereby decreasing the power consumption. The less power consumption at higher value of $\mathrm{h} / \mathrm{d}$ can be also attributed to the fact that blades are exposed to the air. Exposure of blades decreases the drag
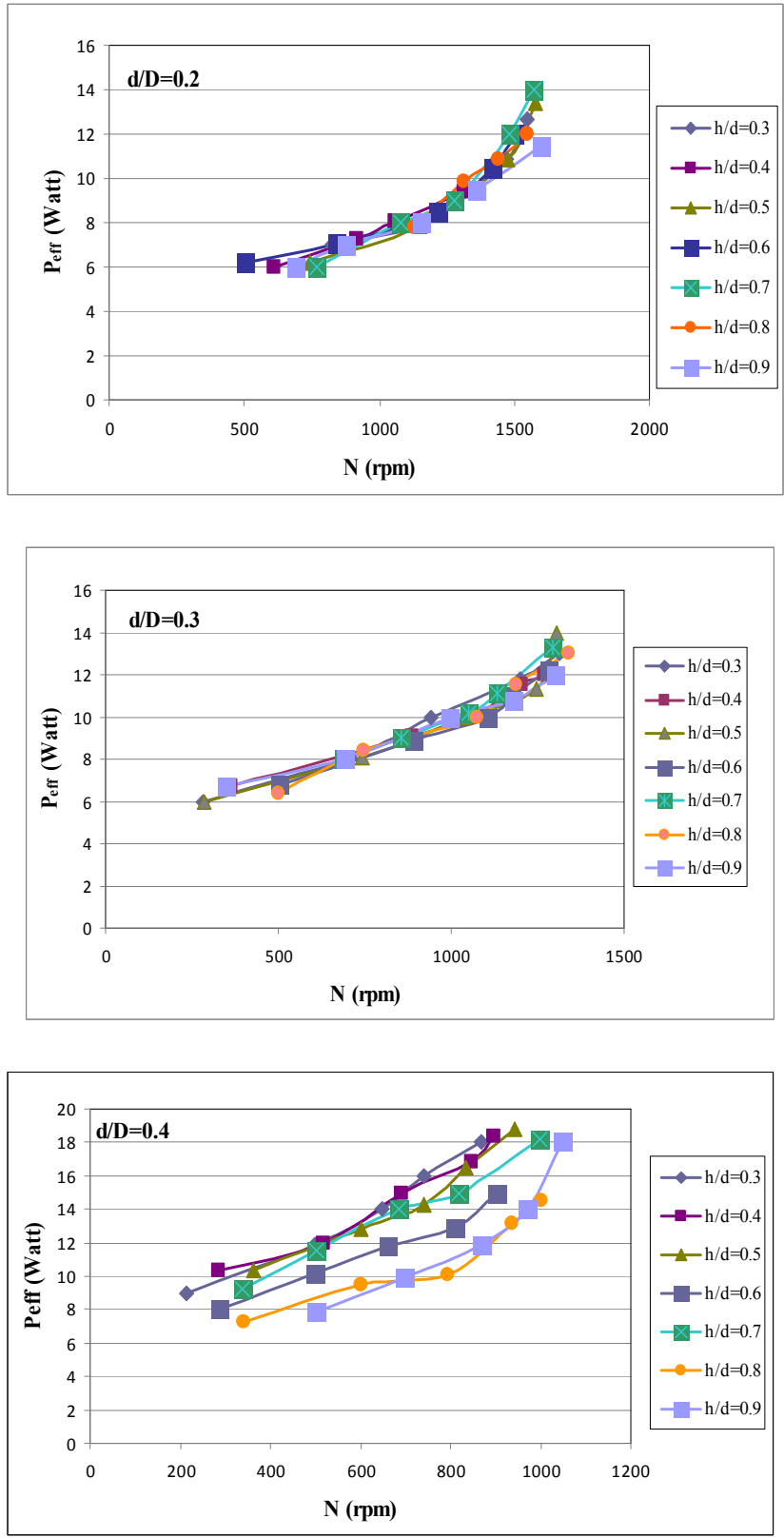

Figure 3b. Influence of submergence depth at power consumption (150 $\mathrm{mm}$ tank) 

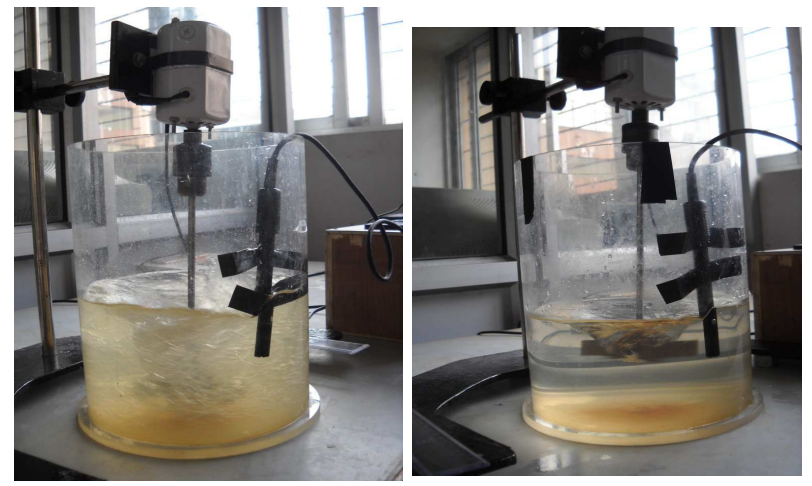

Figure 4. Vortex height with impeller submergence $(\mathrm{h} / \mathrm{d}=0.3$ and 0.8$)$

resistance. This decrease in drag results in lower power draw or consumption. This fact can be seen from the Fig. 3. It can be seen from the Fig. 3, the value of $h / d=0.9$ consumes less power in most of the cases. Overall these observations suggest that a range of 0.7 to 0.9 is optimal as far as power consumption is concerned.

With increasing submergence, the number of eddies at gas-liquid interface are decreased. A decrease in impeller submergence results in stronger surface waves and/or forceful liquid jets. However, the liquid circulation velocity increases with increasing submergence. The centrifugal force due to the strong tangential velocity is the cause for jet formation from the impeller. A vortex is also formed as shown in the Fig. 4. The vortex size increases as the impeller approaches the liquid surface.

The combined effect of bubble entrapment, vortex size and liquid circulation results in a maximum value of oxygen transfer as shown in the Fig. 5. At low submergence depth, momentum of the impeller is not transferred properly to the liquid. In this case, overall liquid velocity is low and thus reducing the oxygen transfer rates. Fig. 5 also suggests that range of 0.7 to 0.9 is optimal for oxygen transfer process.

\section{Conclusion}

Generally standard guidelines for submergence are being followed for designing the stirred tanks for desired process, which can far from being an optimal point. Slight change in submergence depth can cost much when optimal performance is required. There is practically no published information, which explicitly stipulates the optimal submergence depth of impeller.

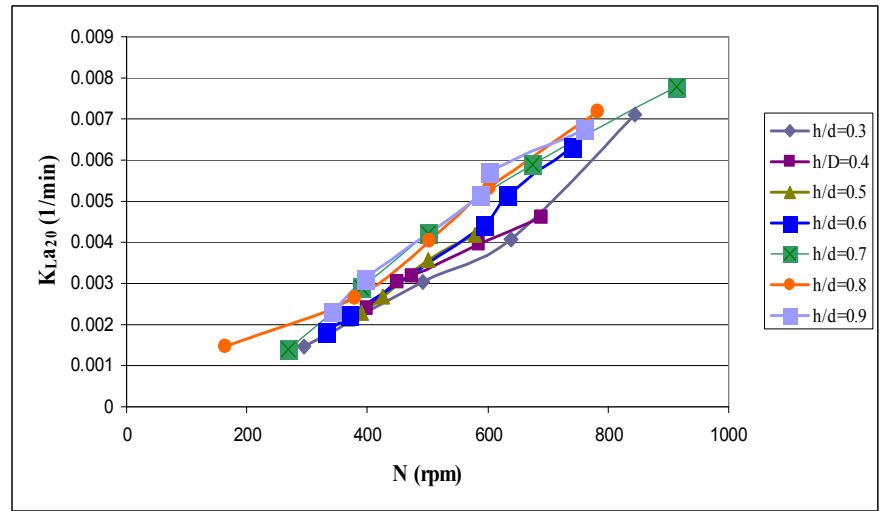

Figure 5. Oxygen transfer at different submergence depth

Present work experimentally investigates the influence of submergence depth on power consumption and oxygen transfer process. The range of $h / d=0.7$ to 0.9 is found to be optimal, however for design purpose $\mathrm{h} / \mathrm{d}=0.9$ can be taken as optimal point.

\section{Acknowledgments}

This work was supported by a research grant from Indian Institute of Technology Guwahati, Guwahati (SG/CE/P/BK/01)

\section{References}

[1] Oldshue, J.Y. 1983. Fluid Mixing Technology. McGraw-Hill, New York.

[2] Ulbrecht, J.J., Patterson, G.K. 1985. Mixing of Liquids by Mechanical Agitation. Chemical Engineering Series: Concepts and Reviews, Volume 1, Gordon and Breach Science Publishers, London.

[3] Assirelli, M., Bujalski, W., Eaglesham, A., Nienow, A.W. 2002. Study of micromixing in a stirred tank using a Rushton turbine: comparison of feed position and other mixing devices. Trans. IChem. 80: 855-863.

[4] Ciofalo, M., Brucato, A., Grisafi, F., Torraca, N. 1996. Turbulent flow in closed and free-surface unbaffled tanks. Chem. Eng. Sci. 51: 3557-3573.

[5] Li, M., White, G., Wilkinson, D., Roberts, K.J. 2004. LDA measurements and CFD modeling of a stirred vessel with a retreat curve impeller. Ind Eng Chem Res. 43: 6534-6547.

[6] Montante, G., Lee, K.C., Brucato, A., Yianneskis, M. 2001. Numerical simulations of the dependency of flow pattern on impeller clearance in stirred vessels. Chem. Eng. Sci. 56: 3751-3770.

[7] Nagata, S. 1975. Mixing Principles and applications. John Wiley \& sons, New York.

[8] Nienow, A.W. 1998. Hydrodynamics of stirred bioreactors. Appl. Mech. Rev. 51: 1-32. 
[9] Ognean, T. 1993. Dimensionless criteria for estimating oxygen transfer in aeration systems. Biotechnol. Bioeng. 41: 1014-1020.

[10] Patil, S.S., Deshmukh, N.A., Joshi, J.B. 2004. Masstransfer characteristics of surface aerators and gasinducing impellers. Ind. Eng. Chem. Res. 43: 2765-2774.

[11] Rushton, J.H. 1952. Applications of fluid mechanics and similitude to scale-up problems-part1. Chem. Eng. Prog. 48: 33-38.

[12] Kumar, B., Devi, T.T., Patel, A.K., Bhatla, A. 2010. Optimal Geometric Configuration for Power Consumption in Baffled Surface Aeration Tanks. Bull. Chem. React. Eng. Catal. 5 (2): 87 - 93

[13] Zlokarnik, M. 1979. Scale-up of surface aerators for waste water treatment. Adv. Biochem. Eng. 11:157-180.

[14] Backhurst, J.R., Harker, J.H., Kaul, S.N. 1998. The performance of pilot and full-scale vertical shaft aerators. Water Res. 22: 1239-1243.

[15] Takase, H., Unno, H., Akehata, T. 1984. Oxygen transfer in a surface aeration tank with square cross section. Int. Chem. Eng. 21: 128-134.

[16] Hwang, H.J., Stenstrom, M.K. 1985. Evaluation of finebubble alpha factors in near-full scale equipment. $J$. Water Pollution Control Fed. 57 : 1142-1150.

[17] Vasel, J.L. 1988. Contribution á l'étude des transferts d'oxygène en gestion des eaux. Ph.D. Thesis, Fondation Universitaire Luxemourgeoise, Luxembourg, Arlon.
[18] Johnson, A.I., Huang, C. 1956. Mass transfer studies in an agitated vessel. AIChE J. 2: 412-419.

[19] Walas, S.M. 1980. Chemical Process Equipment Selection and Design. Butterworth-Heinemann, Washington Street, Newton, MA, USA.

[20] King, R.L., Hiller, R.A., Tatterson, G.B. 1988. Power consumption in a mixer. AIChE J. 34: 506-509.

[21] Ascanio, G., Castro, B., Galindo, E. 2004. Measurement of power consumption in stirred vessels-a review. Chem. Eng. Res. Des. 82: 1282-290.

[22] Nienow, A.W., Ulbrecht, J.J. 1985. Mixing of Liquids by Mechanical Agitation. Eds. Ulbrecht, J.J. and Patterson, G.K., Gordon and Breach, New York, Chapter 6, 203237.

[23] Lewis, W.K., Whitman, W.G. 1924. Principles of gas absorption. Ind. Eng. Chem. 16: 1215-1220.

[24] Brown, L.C., Baillod, C.R. 1982. Modeling and interpreting oxygen transfer data. J. Environ. Eng. 108: 607-628.

[25] Deshmukh, N.A., Joshi, J.B. 2006. Surface aerators: power number, mass transfer coefficient, gas hold up profiles and flow patterns. Chem. Eng. Res. Des. 84: 116. 